\title{
Specific Peculiarities of Conducting Psychological Actions with Oncology Patients
}

\author{
IJCRR \\ Section: Healthcare \\ Sci. Journal Impact \\ Factor: $6.1(2018)$ \\ ICV: 90.90 (2018)
}

\section{Urazbaeva Dilbar Abdullaevna}

Associate Professor, Department of Psychology, Urgench State University, Urgench, Uzbekistan.

\section{ABSTRACT}

Introduction: Nowadays, all over the world, a trend of steady growth in oncological diseases is increasing. This article highlights some problems of onco-psychology and provides an experimental analysis of the characteristics of the disease response and the assessment of the social status of their sickness in patients diagnosed with the oncological illness.

Aim and Objective: The study of women's attitudes towards the disease in patients treated for breast cancer is important in activities aimed at improving the patient's various pain and quality of life.

Method: The study involved 62 female patients diagnosed with breast cancer treated at the Republican Specialized ScientificPractical Medical Center of Oncology and Radiology. The "Serdyuk" questionnaire was used to assess the social status of the disease in patients.

Result: According to the results of the conducted study, the indicators on the assessment of the personal and social status of patients on all scales of the test increased from the bottom up in the stages of the disease. That is, as the stages of the disease progress, they have felt weak, powerless, experienced changes in family relationships, increased tendency to depression, subdepression and depression, decreased leisure time, increased time spent mainly on treatment.

Conclusion: Hospitalization, limited opportunities to improve professional status in society, and as well patients are concerned about looking ugly and unattractive to those around them, especially their spouse, are mentally unwilling, have increased feelings of weakness, felt limited in communication, and experienced economic hardship.

Key Words: Oncology, Psychiatry, Oncology patient, Breast cancer, Emotional state

\section{INTRODUCTION}

Nowadays, all over the world, a trend of steady growth in oncological diseases is increasing. In terms of detection and mortality indicators, oncological diseases rank second only to cardiovascular diseases as the most common cancer in the world (it accounts for $26 \%$ of all deaths). According to a study by the International Agency for Research on Cancer (IARC), by 2030, the number of registered cancer cases will reach 22.2 million. Between the periods 2007 and 2017, the incidence of oncological diseases increased by $33 \%$ (with less growth observed in economically developed countries)..$^{1,2}$

According to statistics, in 2012, the disease was 14.1 million worldwide, the death rate was 8.2 million, and in 2018, 18.1 million patients were registered with the tumour and can- cer types, and $9.6 \mathrm{mln}$ people died worldwide. The patient accounted for several types of tumours and cancer, and 9.6 people died. All over the world, one in six deaths is due to cancer. The most common types of cancer are lung cancer, breast cancer and intestines cancer. ${ }^{3,4}$

The concept of oncology is a general term for a large group of diseases that affect any part of the body. Other terms are also used to describe them: malignant tumours and newly emerging tumours. Symptoms of cancer include rapid growth of abnormal cells, enlargement beyond their normal range, and damage to nearby parts of the body and spread to other organs; this process is called metastases. Metastases are one of the leading causes of death from cancer. 5,6

Psychotherapeutic actions are recognized as one of the most effective treatments, in the World Health System. The impor-

\section{Corresponding Author:}

Dr. Urazbaeva Dilbar Abdullaevna, Associate Professor, Department of Psychology, Urgench State University, Urgench, Uzbekistan; Email: urazbayeva.dilbar@mail.ru

ISSN: 2231-2196 (Print)

Received: 18.06 .2020
ISSN: 0975-5241 (Online)

Revised: 20.07 .2020
Accepted: 07.08 .2020
Published: 22.08 .2020 
tance of the role of the human psyche in the origin, course and treatment of cancer as a result of scientific advances and interdisciplinary integration is reflected in research conducted in the second half of the XX century.

Nevertheless, emphasizing that all diseases cause changes in the human psyche, it should be noted that the internal psychological appearance of oncological diseases is unique, that is, cancer differs from other diseases not only in the physiological state of the patient but also in the psychological state, anxiety, fear, depression and internal anxiety. This situation also indicates the need for a separate psychological study of this category of patients. ${ }^{8,9}$

\section{MATERIALS AND METHODS}

For developing the psychological service in oncology, the psychodiagnostic study was performed with 62 people, diagnosed with breast cancer, of which 16 patients were diagnosed with the first stage (T1N0M0), 18 patients with the second stage (T2N0M0), 16 patients with the third stage (T3N0M0) and 12 patients with the fourth stage (T4NOM0).

The study aims to identify and improve the direction of psychotherapy by studying the attitudes of cancer patients to their disease and its social assessment.

The scientific and methodological basis of the research consisted of the work of Russian scientists (R.A. Luria, V.V. Nikolaeva) on the internal picture of the disease and the impact of severe somatic diseases on the psyche, as well the ideas on the theory of relationships (V.N. Myasishchev).

The concept of "the inner picture of the disease" was introduced into science by the famous Russian scientist R.A. Luria (1935). The author, on the one hand, sought to confirm the subjectivism of man's perception of his disease, its risks and consequences, on the other hand, focused on the effect that the psychological attitude to the disease affects the course of the disease. ${ }^{9}$ R.A. Luria called for caution in assessing patients complaints, discussing their illness with the patients, otherwise the patient's perception of the doctor's words, expressed in vague, subjective emotions, would worsen his condition and even cause mental disorders (iatrogenic). The internal picture of the disease is studied using special medical methods and types of equipment, the set of objective views contrasts with the alloplastic picture of the disorder. ${ }^{8,9}$

The internal appearance of the disease is determined by:

- in the disease nature;

- in its transition stages;

- by type of person (temperament, hierarchy of needs, a typical set of psychological protection, locus control);

- in the state of intellect and brain;

by age;
- concerning this disease in an important microenvironment;

- in the course of the disease. ${ }^{3,4,5}$

V.N.Nikolaeva suggests to divide the system of internal (subjective) picture of the disease into the following levels:

1) sensitive (painful sensations and conditions);

2) emotional (direct emotional reactions to pain sensations and emotional reactions to the consequences of the disease in human life);

3) intellectual (knowledge of the disease and assessment of their condition);

4) motivational (emergence of new motives and reconstruction of the previous motivational system).$^{8}$

A person's attitude towards the disease cannot be viewed in isolation from his or her attitude towards health. Accordingly, an analysis of the internal health landscape is necessary to assess the patient's position. It is also necessary to consider the sensitive, emotional, intellectual and behavioural aspects of this concept. In the choice of diagnostic methods by each specialist, of course, all this requires the need to conduct a study of the internal picture of the disease and the patient's attitude to the disease. One of the important features of a harmonious attitude to the disease is that at the moment of illness a person's behaviour must not disrupt the lives of other people (relatives, colleagues, friends, doctors), must not prevent them from helping the patient.$^{8,10}$

The internal picture of the disease and the attitude to the disease have been studied in the research of Russian scientists P. Zotov, S. Ivanov, A. Tkhostov, G. Arina .1,10

We also based our research on V.N. Myasishchev's theory of relations as a methodological basis. It is known that V.N. Myasishchev (1892-1973) is a Russian psychologist, author of the concept of personality relations. ${ }^{13}$

According to this concept, the basis of an individual is his system of relations with the world around him and with himself. The system of relations arises in the human mind as a result of the reflection of the surrounding reality, which in itself is one of the forms of reflection of reality.

V.N. Myasishchev concludes that activity itself can be a neutral process in the formation of basic mental qualities, without compromising the role of activity in human development, without cooperation between people, relationships that require mutual assistance. ${ }^{7}$

According to V.N. Myasishchev, the relationship arises where the subject and the object exist. A person's psychological relationship represents his or her conscious relationship with objective reality. V.N. Myasishchev distinguished 3 categories of this reality:

1) natural phenomena and the world of objects;

2) people and social events;

3) the subject itself. 
Perception of nature is in many ways subject to the influence of social experience, a person's attitude towards himself is determined by his attitude towards others and the attitude of others towards him. Therefore, in the system of psychological relations, V.N. Myasishchev believes that a person's relationship with other people is of paramount importance. According to V.N. Myasishchev, the types of human attitudes to the environment include needs, emotions, interests and beliefs. Thus, according to his theoretical views, V.N. Myasishchev puts forward the idea that the mental development of man depends on the complexity and expansion of his environment and, above all, the system of relations with other people. ${ }^{711}$ Therefore, the establishment of a trusting relationship with patients diagnosed with oncological disease leads to a positive change in their attitude to the disease, to others, to themselves. This gives significant positive results in influencing the patient's emotional area. ${ }^{12}$

At the diagnostic stage of the study, we used the questionnaire "Assessment of the Social Significance of the Disease" developed by A.I.Serdyuk. This survey determines the level of subjective perception of the impact of the somatic disease on the patient's lifestyle. The methodology is also part of the patients' self-assessment of the "Internal Picture of the Disease" and an important point of psychotherapeutic effect. The methodology was conducted in 1994 by A.I. Serdyuk in 2000 patients with clearly manifested psychosomatic diseases. ${ }^{12,13}$

The test consisted of 10 scales: limited strength and power sense (LS), the patient's deterioration in family relationships (DF), limited enjoyment (LE), deterioration of the patient's relationship at work (DW), limited leisure (LL), career limit (CL), a decrease in physical attractiveness (DP), the formation of a sense of weakness (SW), limited communication (LC), and financial damage (FD). ${ }^{14}$

In this survey, patients' self-assessment is considered to be part of the "internal picture of the disease" and an important point of psychotherapeutic impact.

\section{RESULTS AND DISCUSSION}

Below the results of the survey are given. The stage of the disease was interpreted for each criterion (figure 1).

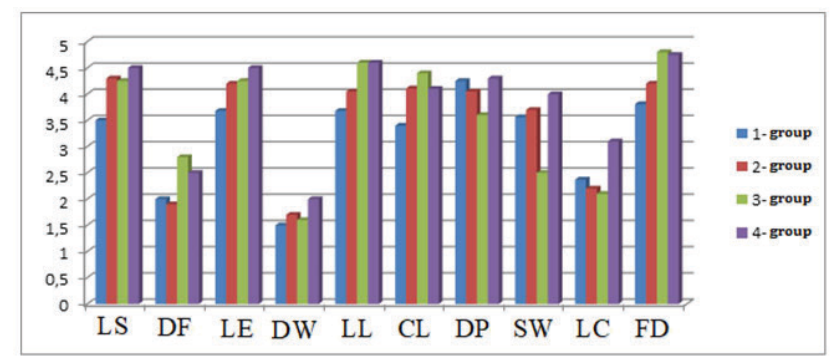

Figure 1: The appearance of personal attitudes of patients in different stages of the disease.
Note: LS - limited strength and power sense, DF- the patient's deterioration in family relationships, LE- limited enjoyment, DW - deterioration of the patient's relationship at work, LL- limited leisure, CL- career limit, DP- a decrease in physical attractiveness, SW- the formation of a sense of weakness, LC- limited communication, FD- financial damage.

As can be seen from the histogram above (Figure 1), according to the results of the Serdyuk survey obtained in each group of patients, we can see that there are significant changes in the characteristics of patients' relationships due to the disease. In particular, on the scale of feeling the limit of weakness, we can see that the arithmetic means in the first stage of the disease is 3.5 , in the second stage 4.3 , in the third stage 4.25 , and the fourth stage 4.5 . That is, it can be acknowledged that feeling weak and powerless due to the disease increases as the stages of the disease increase. The effects of the disease on the body also have a direct effect on the psyche of the individual.

On the scale of patient relationship deterioration in the family, we can see that the arithmetic means in the first stage of the disease was 2 , in the second stage 1.9 , in the third stage 2.8 , and the fourth group 2.5. Accordingly, in groups 1 and 3, we can see an increase in conflict situations in the patient's family relationships with their relatives about the previous groups.

According to the results of the test obtained on the scale of patient pleasure limit, the arithmetic mean in patients with the first stage of the disease was 3.68, in the second stage -4.2 , in the third stage -4.25 , in the fourth stage -4.5 . It is self-evident that the disease harms the patient's psyche, the sphere of pleasure. This is precise because of the low treatment expectations for future treatment options and overall recovery from the disease, as the incidence of the disease increases with developing susceptibility to degradation, subdepression, and depression.

In the study, we can see that patients' attitudes deteriorated on a scale of relative elevation in the second and fourth stages. It was the lowest rate, i.e., the incidence of relationship deterioration at work, which was shown to be much lower than on other scales at all stages. This may be due to the fact that patients are more relieved in the workplace after they become ill, the relationship with patients is warmer in relation to colleagues at work, and the situation is relatively relieved.

On the scale of leisure time constraints, we can see that as the stages of the disease increase, the amount of leisure time decreases, mainly the time spent on treatment, hospitalization increases. The arithmetic mean of the results obtained on this scale was 3.68 in the first stage, 4.05 in the second stage, 4.6 in the third stage, and 4.6 in the fourth stage. An upward trend was observed from the first stage to the upper stages.

There was an increase from the first stage of the disease to stage 3 on the scale of limited opportunities for professional 
advancement, and a relative decrease in the fourth stage. That is, the feeling that a person is unable to work actively in society due to the disease, to work wherever he wants, to have good jobs, to increase his professional status, increased from the first stage of the disease $(3,4)$ to the third stage $(4,4)$. The arithmetic mean of the results obtained in the fourth stage fell to 4.1. From this, it can be concluded that the anxiety of not being able to have a place in society due to illness will rise to level 3. In the fourth stage, the acceptance of the disease affects the goals associated with the possibility of increasing professional status in society.

The arithmetic mean of the results from the scale associated with the decrease in physical attractiveness was 4.25 in the first stage of the disease, 4.05 in the second stage, 3.6 in the third stage, and 4.3 in the fourth stage. That is, medical treatments for the disease include surgery (partial or complete removal of the breast-mastectomy), chemotherapy, hair loss as a result of light therapy, skin discolouration, etc., which is a very big crisis, especially for a woman, loss. In particular, patients' concern that they would look ugly and unattractive to those around them, especially their spouse, was evident in the first and fourth stages of the disease. Because for a woman, the "symbol of femininity" and "feminine beauty" are defined by the breasts, gynaecological (reproductive) area, hair, eyebrows and eyelashes. Breast mastectomy, partial or complete removal of the breast, hair loss as a result of chemotherapy, causes a decrease in its external attractiveness in sick women, anxiety from ugliness, anxiety and a feeling of inferiority.

The arithmetic mean of the results obtained for experiencing mental unwillingness, weakness, feelings of unhappiness due to illness was shown to be 3.56 in the first stage, 3.7 in the second stage, 2.5 in the third stage, and 4 in the fourth stage. In the first, second, and fourth stages of the disease, an increase in the feeling of weakness was observed, and in the third stage, a feeling of relative indifference was observed.

According to the results of tests on interpersonal relationships due to the disease, difficulty in communication, feeling of limited communication, the arithmetic mean was 2.37 in the first stage, 2.2 in the second stage, 2.1 in the third stage. In the 4th stage, we can see that it has risen to 4 , that is, the communication is limited and difficult.

The significant financial damage caused by the disease was associated with an increase in the stages of the disease, an increase in economic damage (1-3.81, 2-4.2, 3-4.8, 4-4, using a scale that determines whether the treatment process caused economic hardship). 75). Only in the fourth stage did this figure decline relatively insignificantly. Because this stage is the last stage of the disease, it is often characterized by metastasis (cell growth, damage to other organs), enlargement of lymph nodes, the inability of the body to tolerate drugs that cause high economic damage.

\section{CONCLUSION}

Thus, according to the results of the study, the indicators for assessing the personal and social status of patients on all scales increased from the bottom up in the stages of the disease. That is, as the stages of the disease progress, they have felt weak, powerless, experienced changes in family relationships, increased tendency to depression, sub-depression and depression, decreased leisure time, increased time spent mainly on treatment, hospitalization, limited opportunities to improve professional status in society, and as well patients are concerned about looking ugly and unattractive to those around them, especially their spouse, are mentally unwilling, have increased feelings of weakness, felt limited in communication, and experienced economic hardship.

In conclusion, the choice of psychotherapy in psychotherapy with oncology patients should be based on the nature of the patient's attitude to the disease and the assessment of his social status.

\section{Acknowledgement: None}

Conflict of interest: None

Source of Funding: None

\section{REFERENCES}

1. Zotov P.B., Umansky S.M. The internal picture of the disease of cancer patients, Materials of the 1st All-Russian Congress "Mental Disorders in Oncology" Abstracts. report (2008); 9-20.

2. Karvasarsky B.D. Clinical Psychology: Textbook. - 2nd ed. Sanct Petersburg: Peter, 2006;960 -965.

3. Kosyrev VN, Ushakov GK. Clinical psychology: Textbook. the method, complex for teachers and students of psychology faculties. Med. psyc. 1984; 2(10): 272 -275.

4. Lichko A.E. Internal picture of the disease. - Chisinau: Sci. 1980; 235-242.

5. Malkina-Pykh IG, Psychosomatics: A Practical Psychologist's Handbook. Eskimo, 2005; 992-995.

6. Myasishchev V. N. Personality and neuroses. Publishing house of LSU, 1960; 150-160.

7. Nikolaeva V.V. The influence of chronic illness on the psyche. - M.: Publishing house of Moscow State University, 1987; 2(4):153-159.

8. Romek V.G. Behavioral psychotherapy: Textbook. manual for university students. Acad. 2002; 4(6):192 -199.

9. Tkhostov A. Sh, Arina G.A. Theoretical problems of the study of the internal picture of the disease, Psychological diagnosis of attitudes towards the disease.- L., 1990;3:56-61.

10. http://www.oncology.ru/news/2019/10/14/, Global cancer research news and achievements. 2019.

11. Global Cancer Statistics 2018: GLOBOCAN Estimates of Incidence and Mortality Worldwide for 36 Cancers in 185 Countries. Bray F, et al. CA: A Cancer Journal for Clinicians. https:// onlinelibrary.wiley.com/doi/full/10.3322/caac.21492. 2018

12. Fact sheets: the World Health Organisation. https://www.who. $\mathrm{int} / \mathrm{ru} /$ news-room/fact-sheets/detail/cancer. 2017. 